\title{
Homogalacturonan deesterification during pollen-ovule interaction in Larix decidua Mill.: an immunocytochemical study
}

\author{
Katarzyna Rafińska • Michał Świdziński • \\ Elżbieta Bednarska-Kozakiewicz
}

Received: 17 October 2013 / Accepted: 1 April 2014 / Published online: 4 May 2014

(C) The Author(s) 2014. This article is published with open access at Springerlink.com

\begin{abstract}
Studies on angiosperm plants have shown that homogalacturonan present in the extracellular matrix of pistils plays an important role in the interaction with the male gametophyte. However, in gymnosperms, knowledge on the participation of HG in the pollenovule interaction is limited, and only a few studies on male gametophytes have been reported. Thus, the aim of this study was to determine the distribution of HG in male gametophytes and ovules during their interaction in Larix decidua Mill. The distribution of HG in pollen grains and unpollinated and pollinated ovules was investigated by immunofluorescence techniques using monoclonal antibodies that recognise high methyl-esterified HG (JIM7), low methyl-esterified HG (JIM5) and calcium cross-linked HG (2F4). All studied categories of HG were detected in the ovule. Highly methyl-esterified HG was present in the cell walls of all cells throughout the interaction; however, the distribution of low methylesterified and calcium cross-linked HG changed during the course of interaction. Both of these categories of HG appeared only in the apoplast and the extracellular matrix of the ovule tissues, which interact with the male gametophyte. This finding suggests that in L. decidua, low methyl-esterified and calcium cross-linked HG play an important role in pollen-ovule interaction. The last category of HG is most likely involved in adhesion between the pollen and the ovule and might provide an optimal calcium environment for pollen grain germination and pollen tube growth.
\end{abstract}

K. Rafińska · M. Świdziński · E. Bednarska-Kozakiewicz ( $\square)$ Department of Cell Biology, Faculty of Biology and Environment Protection, Nicolaus Copernicus University, Lwowska 1, 87-100 Toruń, Poland

e-mail: ebedn@umk.pl
Keywords Adhesion · Extracellular matrix · Gymnosperms · Pollen grain · Pollen tube growth · Pollination

$\begin{array}{ll}\text { Abbreviations } \\ \text { HG } & \begin{array}{l}\text { Homogalacturonan (homopolymer of } \alpha \text {-1,4-linked } \\ \text { galacturonic acid) }\end{array} \\ \text { PMEs } & \text { Pectinmethylesterases } \\ \text { RT } & \text { Room temperature } \\ \text { PG } & \text { Polyethylene glycol } \\ \text { PBS } & \text { Phosphate-buffered saline } \\ \text { BSA } & \text { Bovine serum albumin } \\ \text { DAPI } & 4^{\prime}, 6 \text {-Diamidino-2-phenylindole } \\ \text { ecm } & \text { Extracellular matrix }\end{array}$

\section{Introduction}

In seed plants, the extracellular matrix of female reproductive organs is where interaction between ovules and the male gametophyte occurs. In angiosperms, pollen grains germinate on the stigma, and the pollen tube continues its growth towards the female gametophyte in the ecm of the style. In gymnosperm plants, unlike in flowering plants, pollen grains land directly on the ovules; therefore, the male gametophyte interacts only with the ecm secreted by the ovule tissues. However, in both angiosperms and gymnosperms, ecm of the female reproductive organs should provide specific ions and molecules necessary for nutrition, attraction and guidance of the male gametophyte. Many studies in angiosperms have shown that the pollen-pistil interaction involves such ecm components as homogalacturonan, arabinogalactan proteins and calcium ions (Cheung et al. 1995; Jauh and Lord 1996; Mollet et al. 2000; Lenartowska et al. 2001; Zhao et al. 2004; Bednarska et al. 2005; 
Hristova et al. 2005; Coimbra et al. 2007; Ge et al. 2009; Sage et al. 2009; Costa et al. 2013).

In angiosperms, $\mathrm{Ca}^{2+}$ plays a particularly important role in pollen grain germination and pollen tube growth. These ions are taken up by germinating pollen grains and growing pollen tubes forming a specific tip-to-base $\mathrm{Ca}^{2+}$ gradient. Elevated levels of $\mathrm{Ca}^{2+}$ at the tube tip are involved in vesicle secretion and in determining the orientation of the tube growth (see the review by Ge et al. 2007; Hepler and Winship 2010). Similarly, in gymnosperms, $\mathrm{Ca}^{2+}$ is taken up by growing pollen tubes (Lazzaro et al. 2005; Chen et al. 2009), and as $\mathrm{Wu}$ et al. (2008) found, the $\mathrm{Ca}^{2+}$ influx in Pinus bungeana is even higher than in angiosperm pollen tubes. The main $\mathrm{Ca}^{2+}$ store in the ecm of plant cells is $\mathrm{HG}$, which is the most abundant pectic polysaccharide (see the review by Wolf et al. 2009). HG is synthesised and methylesterified in the Golgi apparatus.

Within the cell wall, high methyl-esterified HG can undergo deesterification by PMEs. These enzymes remove the methyl groups from the HG chain leading to the formation of free carboxyl groups and to the release of methanol and protons. Free carboxyl groups can bind $\mathrm{Ca}^{2+}$, and a stretch of at least nine deesterified galacturonic acid residues can form an 'egg-box' structure due to the formation of $\mathrm{Ca}^{2+}$ cross-bridges. The 'egg-box' structures participate in gel formation and, thus, strengthen the cell wall; they can also become a target for pectin-hydrolysing enzymes, such as polygalacturonases and pectin/pectate lyases (see the review by Wolf et al. 2009). The action of PMEs is influenced by a range of factors, including cell wall $\mathrm{pH}$ and the pattern of methyl-esterification of HG chains.

Deesterification of HG is a process that plays a significant role in the pollen-pistil interaction in angiosperms. It has been shown that changes in HG methyl-esterification status during the pollen-pistil interaction depend on the type of pistil. In the unpollinated pistil of Haemanthus albiflos (dry stigma and hollow style), the high
Fig. 2 The cytological view of the pollen-ovule interaction in $L$. decidua. a A mature pollen grain containing tube cell, generative cell, sterile cell and two degenerated prothallial cell. b The strong vacuolated cells of the unpollinated stigmatic tip (stage of megasporocyte). c The degenerating cells of the pollinated stigmatic tip (stage of megaspores). d-f The ovule at the stage of the cellular gametophyte with a differentiated central cell. d Pollen grains in the micropylar canal. At the distal end of the canal, fragments of exine shed by the pollen grain are visible. The cellular gametophyte is surrounded by degenerating tapetal cells. e A pollen grain in the micropylar canal filled with secretions. f On the surface of the nucellus, secretions (arrow) are visible. g-h Mature ovule. $\mathbf{g}$ Pollen grain germinating on the surface of the nucellus. $\mathbf{h}$ A pollen tube growing in the nucellus; cells surrounding the pollen tube are degenerated. Ex exine, $G$ gametophyte, $G C$ generative cell, In integument, $M$ stigmatic tip, $M C$ micropylar canal, $N$ nucellus, $n u$ nucleus, $P C$ prothallial cell, $P G$ pollen grain, $P T$ pollen tube, $S$ sporoderm, $S C$ sterile cell, $T$ tapetum, $T C$ tube cell. Scale bars $10 \mu \mathrm{m}$

methyl-esterified HG form was mainly detected (Bednarska et al. 2005; Lenartowska et al. 2011); HG deesterification occurs in the cell walls of the stigma and style during pollen germination and pollen tube growth. In Petunia hybrida and Olea europaea L. (wet stigma and solid style), low methyl-esterified HG was already present in the stigma exudates and ecm of the transmitting tissue during pollination (Lenartowska et al. 2001; Bednarska et al. 2005; Suárez et al. 2013). Additionally, previous studies have indicated that in the transmitting tissue of the P. hybrida pollinated style, lysis of deesterified HG was accompanied by a strong increase in $\mathrm{Ca}^{2+}$ levels in the ecm (Bednarska et al. 2005). Therefore, in the P. hybrida, low-esterified HG can serve as a $\mathrm{Ca}^{2+}$ source for pollen tubes growing in the transmitting tract. In the lily, low-esterified HG together with small $(9 \mathrm{kDa})$ proteins is responsible for the in vivo tube adhesion to the wall surface of the stylar epidermis (Mollet et al. 2000; Park et al. 2000).

The role of the components of the ecm in the sexual reproduction of conifers has not been intensively studied. So far, there are only a few reports on this topic, and they

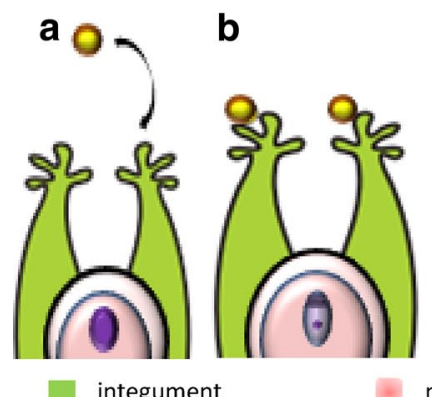

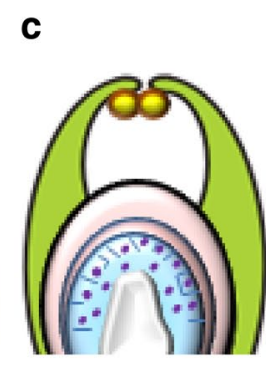

nucellus d

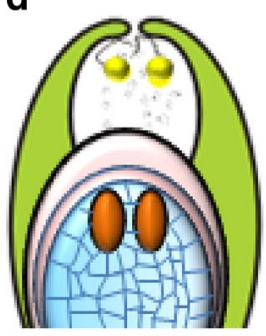

gametophyte

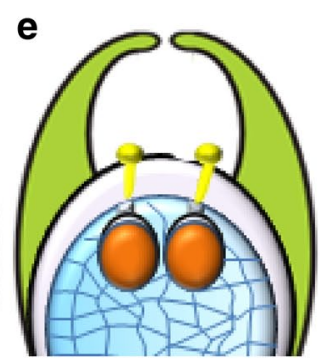

archegonium
Fig. 1 Successive stages of pollen-ovule interaction in L. decida. a The ovule at the megasporocyte stage-period of the pollen shed. b Stage of megaspores-stigmatic tip of the ovule is pollinated. c Free nuclear gametophyte stage-pollen grains are engulfed into the micropylar canal of the ovule. d Cellular gametophyte stage-pollen grains are carried to the nucellar apex. e Mature ovule-pollen tubes penetrate the nucellus 


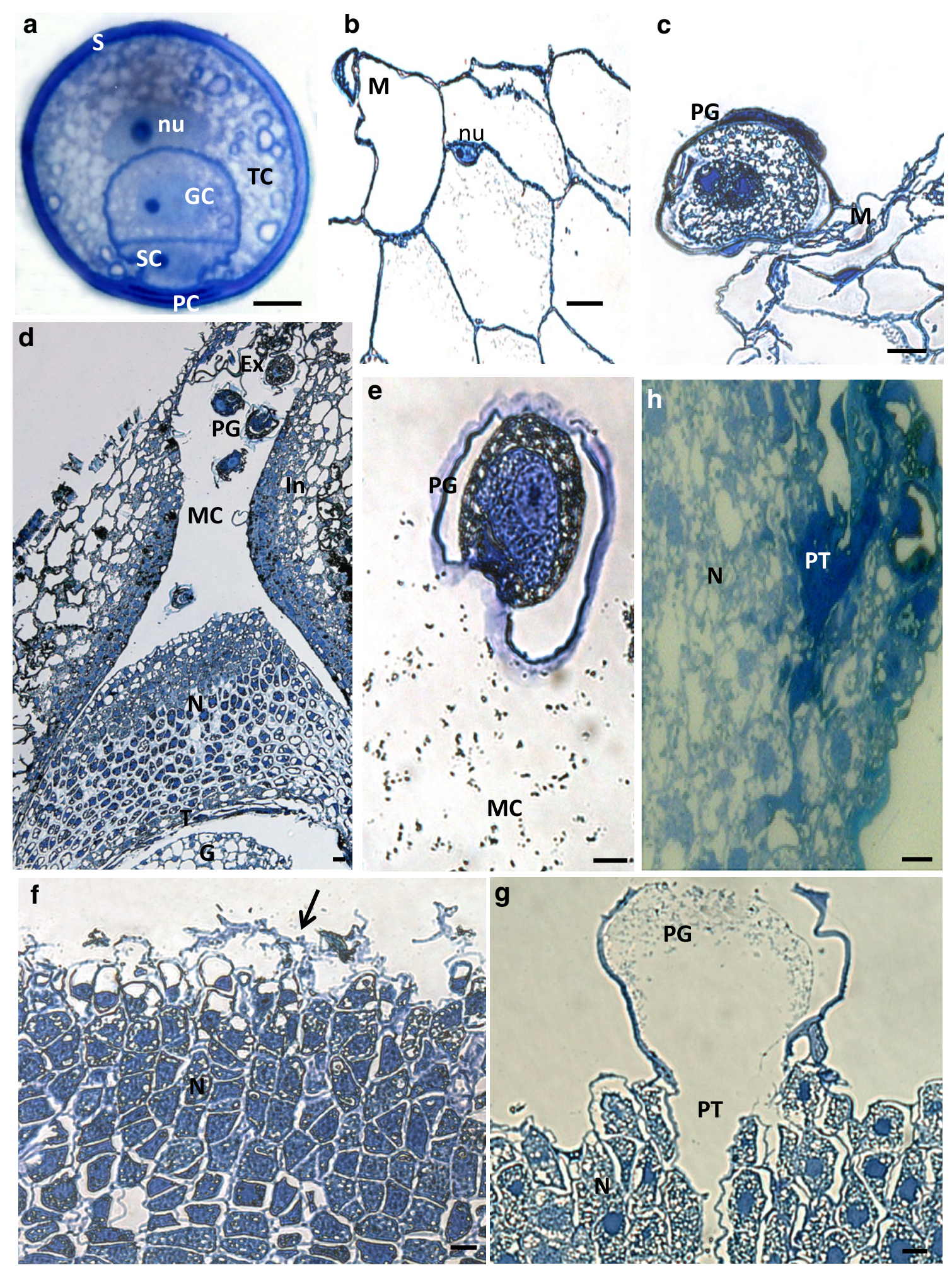

focus primarily on pollen tube wall synthesis (Derksen et al. 1999; Mogami et al. 1999; Chen et al. 2008; Wu et al. 2008). To date, there is practically no data on the nature and role of HG during the interaction between the male gametophyte and ovule of gymnosperm plants. Therefore, the aim of this study was to analyse the distribution of different categories of HG in the male gametophyte and ovules of Larix decidua before and after pollination. The potential role of HG in the sexual processes of gymnosperms is discussed and includes a comparison with available data on 
HG behaviour during pollen-pistil interaction in flowering plants.

\section{Materials and methods}

Plant material

Male and female cones of Larix decidua Mill. were collected from trees growing in the garden of the Faculty of Biology and Environmental Protection, Nicolaus Copernicus University, Toruń, Poland.

\section{Preparation of material}

Mature pollen cones were collected in March and April. They were surface sterilised in $70 \%$ ethanol for $40 \mathrm{~s}$ and then in $10 \%$ sodium hypochlorite. Cones were rinsed in sterile distilled water and dried at RT in sterile Petri dishes covered with sterile filter paper. Before culturing, pollen grains were hydrated for $24 \mathrm{~h}$ at $24{ }^{\circ} \mathrm{C}$ in sterile conditions. Subsequently, the prepared pollen was germinated in the medium contained Brewbaker and Kwack minerals diluted 1:10 supplemented with $18 \%$ PG 4000, $7 \%$ sucrose, $0.4 \%$ phytagel, nystatin $(0.0041 \mathrm{~g} / 25 \mathrm{ml})$ and chloramphenicol $(0.0014 \mathrm{~g} / 25 \mathrm{ml})$, and the $\mathrm{pH}$ was adjusted to 5.2. In this medium, pollen grains were cultured together with sterilised nucelli and archegonia. The cultivation was carried out at $24{ }^{\circ} \mathrm{C}$ in the dark. For immunolocalisation of $\mathrm{HG}$, the pollen tubes were collected after 7 days of growth and fixed in a mixture of $4 \%$ paraformaldehyde and $0.25 \%$ glutaraldehyde in PBS with $\mathrm{pH} 7.2$ for $2 \mathrm{~h}$ at RT. The fixed pollen tubes were individually transferred to cover glasses coated with a drop of poly-L-lysine.

Female cones were collected from March to June. This period encompassed successive stages of the interaction between the male gametophyte and ovule, including pollination, engulfment of pollen grains into the micropylar canal, pollen grain germination and pollen tube growth (Fig. 1). Dissected ovules were fixed in $4 \%$ paraformaldehyde and $0.25 \%$ glutaraldehyde in PBS, placed under vacuum for $1 \mathrm{~h}$ and then held at $4{ }^{\circ} \mathrm{C}$ overnight. Fixation was followed by buffer rinses ( 3 times for $20 \mathrm{~min}$ ). The material was dehydrated in increasing concentrations of ethanol, including several washes in absolute ethanol, and then infiltrated and embedded in BMM resin at $-20{ }^{\circ} \mathrm{C}$ under UV light for polymerisation (butyl methacrylate, methyl methacrylate, benzoin ethyl ether and dithiothreitol from SigmaAldrich). The embedded material was cut on a Leica UCT ultramicrotome into semi-thin serial sections $(1,000 \mathrm{~nm})$ and placed on microscope slides coated with biobond (British Biocell International, Cardiff, UK). Before performing immunocytochemical reactions, the BMM resin was
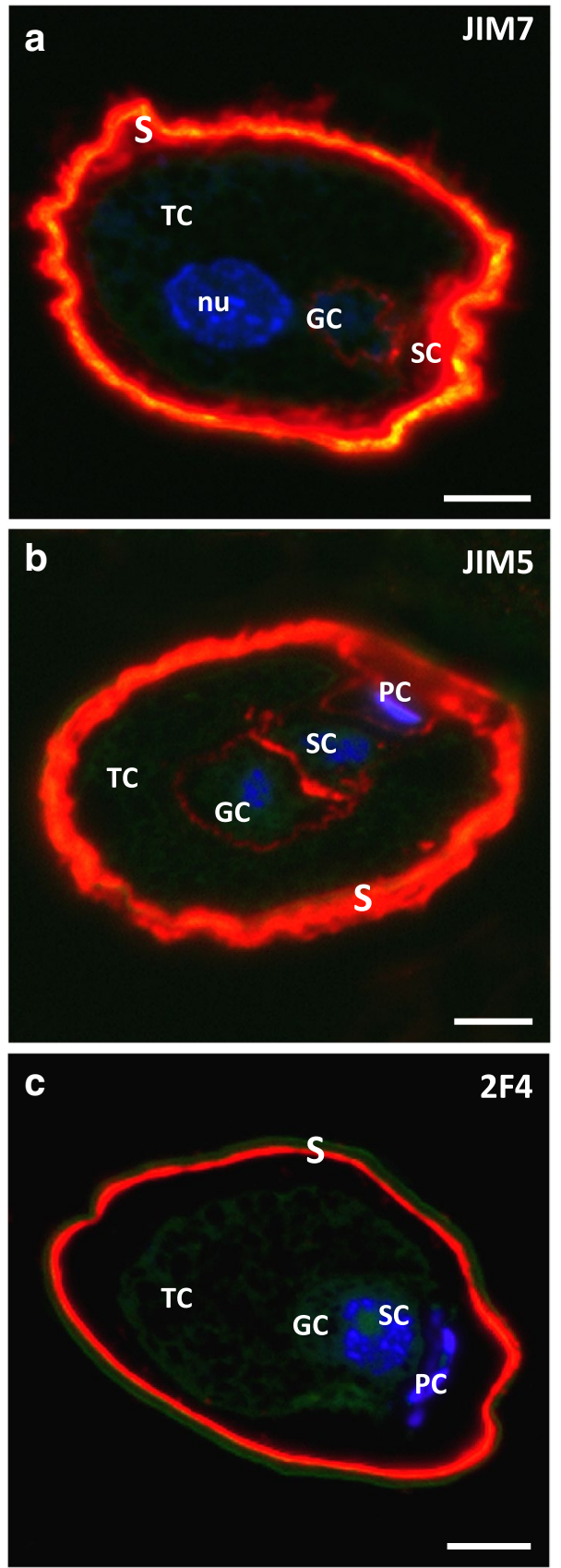

Fig. 3 Immunolocalisation of $\mathrm{HG}$ in the mature larch pollen grain. a An intense JIM7 labelling was present in the pollen wall. Lower fluorescence was visible in the walls of pollen cells. b JIM5 labelled the wall of the pollen grain as well as pollen cells. c 2F4 labelling was found only in the pollen wall. $G C$ generative cell, $n u$ nucleus, $P C$ prothallial cell, $P G$ pollen grain, $S$ pollen wall, $S C$ sterile cell, $T C$ tube cell. Scale bars $10 \mu \mathrm{m}$

removed by washing the slides in pure acetone twice for $10 \mathrm{~min}$. The sections were then washed twice in water and, finally, in PBS.

Pollen grains used for the immunocytochemical reaction were collected from male cones at the time of 

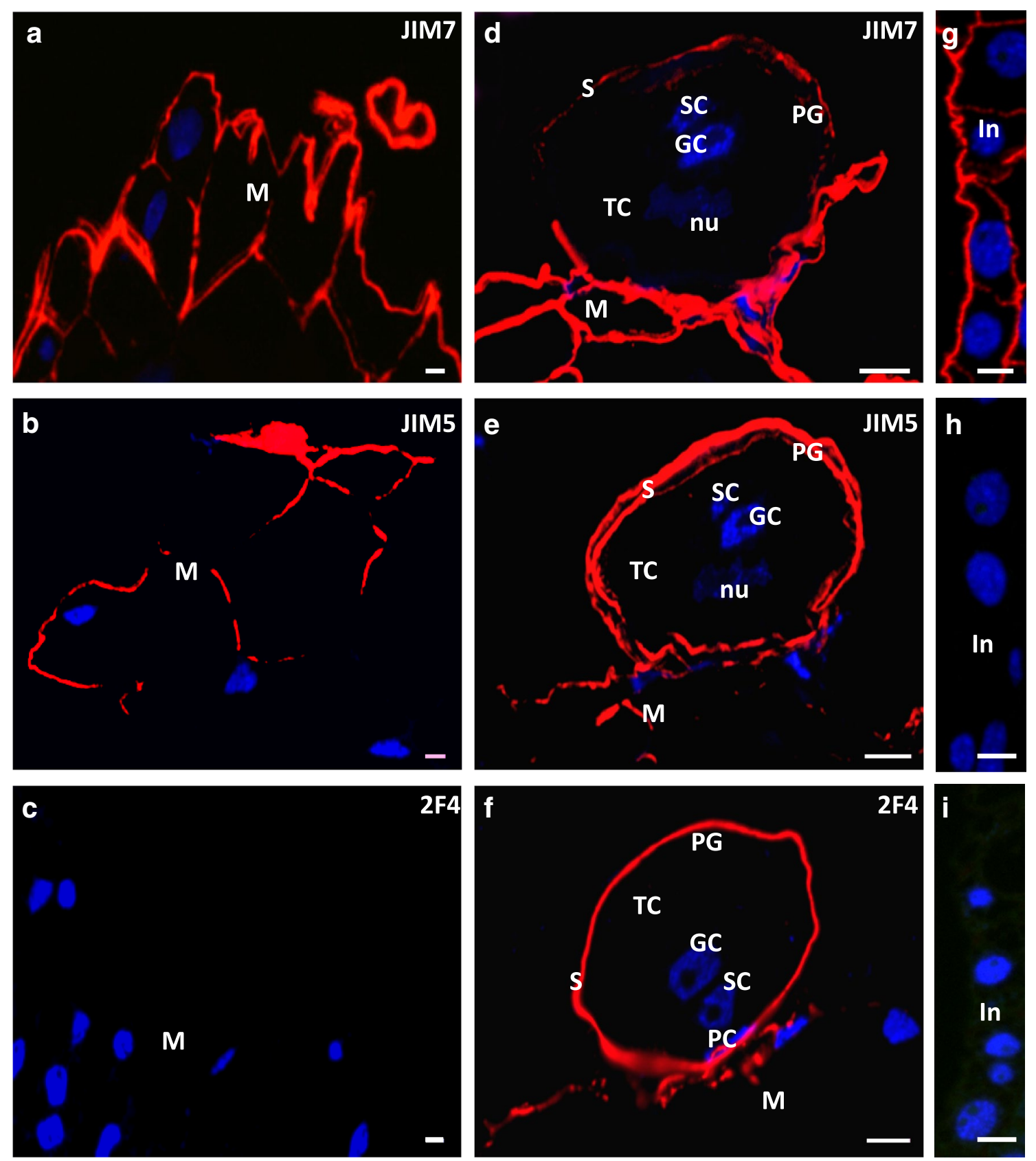

Fig. 4 Immunolocalisation of HG in the unpollinated and pollinated larch ovule. a-c The unpollinated stigmatic tip. a JIM7 labelling was seen in the walls of all cells. b JIM5 labelling was present only in some walls. c 2F4 labelling was not detected. d-f The pollinated stigmatic tip. d For the JIM7Ab, strong signal was present in the walls of the degenerating stigmatic tip cells, and a weaker labelling occurred in the pollen grain wall. e With regard to the JIM5 Ab, a weak fluorescence was observed in the degenerating stigmatic tip, and a strong labelling in the pollen grain wall. $\mathbf{f}$ At the place of pollen grain adhesion, on the surface of the stigmatic tip and in the pollen grain wall, the epitope recognised by $2 \mathrm{~F} 4$ was visible. $\mathbf{g}-\mathbf{i}$ The integument. $\mathbf{g}$ In the cell walls, signal of JIM7 was found. $\mathbf{h}, \mathbf{i}$ The integument was not labelled by JIM5 (h) or 2F4 (i). $G C$ generative cell, In integument, $M$ stigmatic tip, $P C$ prothallial cell, $P G$ pollen grain, $S$ pollen wall, $S C$ sterile cell, $T C$ tube cell. Scale bars $10 \mu \mathrm{m}$

grains were embedded in agar blocks by the use of lukewarm $2 \%$ agar. The material was dehydrated, embedded in BMM resin and cut into semi-thin sections as described previously. 
Immunolocalisations

Three different primary antibodies (JIM7, JIM5 and 2F4) (PlantProbes, Paul Knox Cell Wall Lab., University of Leeds, UK) were used for the immunodetection of cell wall antigens. The JIM7 antibody recognises low-unesterified epitopes of HG but does not bind to fully unesterified HG, the JIM5 antibody-fully unesterified and low methyl-esterified HG (Knox et al. 1990; Verhertruggen et al. 2009) and the 2F4 antibody binds specifically to a dimeric association of deesterified HG chains through calcium ions (Liners and van Cutsem 1992). Immunolocalisation of high-esterified and low-esterified HG was performed as previously described by Rafińska and Bednarska (2011). The sections were treated with blocking solution (PBS with $2 \% \mathrm{BSA}$ ) for $1 \mathrm{~h}$ and then incubated with the JIM7 or JIM5 antibody (diluted 1:50 in PBS with $0.2 \%$ BSA) overnight at $4{ }^{\circ} \mathrm{C}$. After washing with $\mathrm{PBS}$, the sections were incubated with secondary rabbit antirat IgG antibody conjugated with Cy3 (Jackson ImmunoResearch Laboratories, West Baltimore Pike, PA, USA) that was diluted 1:100 in PBS with $0.2 \%$ BSA for $1 \mathrm{~h}$ in the dark. Finally, the sections were washed with PBS.

To detect calcium cross-linked $\mathrm{HG}$, the sections were blocked with $0.5 \%$ BSA in $20 \mathrm{mM}$ Tris- $\mathrm{HCl}$ for $10 \mathrm{~min}$ and incubated with primary antibody $2 \mathrm{~F} 4$ (diluted 1:50 in $0.1 \% \mathrm{BSA}$ in $20 \mathrm{mM}$ Tris- $\mathrm{HCl}$ ) for $1 \mathrm{~h}$ at room temperature. Next, the slides were washed in the same buffer four times and incubated with antimouse IgG secondary antibody labelled with $\mathrm{Cy} 3$ (Sigma-Aldrich) that was diluted 1:50 in $20 \mathrm{mM}$ Tris- $\mathrm{HCl}$ buffer with $0.1 \%$ BSA for $1 \mathrm{~h}$ at RT in the dark. Finally, the samples were washed in Tris-HCl.

DNA in the sections was stained with a water solution (4 pg/ml) of DAPI (Fluka) for $5 \mathrm{~min}$. Then, the sections were washed in distilled water, dried at room temperature and covered with $0.5 \% \mathrm{~N}$-phenylenediamine. Controls were performed omitting the incubation step with the primary antibodies; specific labelling was not observed in all control reactions (cf. Fig. 5j). Observations were performed using a Nikon Eclipse 80i fluorescence microscope. Images were captured by using a Nikon DS$5 \mathrm{Mc}$ colour-cooled digital camera paired with the Lucia G 5.30 image analysis software. The camera settings of exposure time, gain and offset were kept constant. Images of in vitro growing pollen tubes were acquired by the confocal Nikon microscope PCM 2000-Eclipse TE 300 paired with the EZ 2000 Viewer software package. The signals from antibodies were observed as a red fluorescence of $\mathrm{Cy} 3$, and additional images were collected in the green (autofluorescence) and blue (DAPI fluorescence) channels.
Fig. 5 Immunolocalisation of HG in the larch ovule after the engulfment of the pollen grain into the micropylar canal-free nuclear gametophyte stage. a-c The stigmatic tip. a A JIM7, b JIM5, c 2F4 labelling of all studied HG epitopes occurred in the material formed as a result of the degeneration of the stigmatic tip cells. $\mathbf{d}-\mathbf{f}$ The integument. d JIM7 signal was present in the cell walls of all cells of the integument. e JIM5 fluorescence was detected only in the cell walls situated deep within the integument. $f$ The integument was completely devoid of fluorescence from 2F4. g-i The nucellus. g Epitopes recognised by JIM7 were visible in all the cell walls of the nucellar cells. h, i A JIM5 (h) and 2F4 (i) were localised only in some cell walls situated below the surface of the nucellus. $\mathbf{j}$ The negative control showed no labelling in the ovule when the primary rat antibody was omitted. In integument, $M$ stigmatic tip, $M C$ micropylar canal, $N$ nucellus. Scale bars $10 \mu \mathrm{m}$

\section{Results}

Interaction between the male gametophyte and ovule

The successive stages of interaction between the male gametophyte and ovule in larch are schematically shown in Fig. 1 (Rafińska and Bednarska 2011). In L. decidua, pollen is shedding when the megasporogenesis takes place in the ovule (Fig. 1a, b). The pollen grains are non-saccate (Fig. 2a) and land on the expanded surface of the integument called the stigmatic tip (Fig. 1b). The cells of the unpollinated stigmatic tip are strongly vacuolated (Fig. 2b) and collapse after pollination (Fig. 2c). Over several days, pollen grains are engulfed into the micropylar canal of the ovule (Fig. 1c). At this time, a functional megaspore develops in the free nuclear gametophyte (Fig. 1c). At the distal end of the micropylar canal, pollen grains hydrate and shed their exine (Fig. 2d). After gametophyte cellularisation (Fig. 1d), fluid secretions fill the canal, and pollen grains, enclosed only by intine, are carried to the nucellar apex (Fig. 2e). At this time, the cells situated in the nucellar apex are smaller and strongly vacuolated, while the cells in the central region of the nucellus are larger and have thicker walls (Fig. 2d). Cells of the apex collapse and secretion appear on the surface of the nucellus (Fig. 2f). Pollen grains germinate on the nucellar surface (Fig. 2g) when the prothallium contains mature archegonia (Fig. 1e). Within few days, the pollen tubes penetrate the nucellus (Fig. 2h), followed by the neck cells and ventral canal cell, and grow into the egg cell.

Localisation of HG epitopes in the mature pollen grain and unpollinated ovule

In the wall of the mature, hydrated pollen grain, $\mathrm{HG}$ epitopes recognised by JIM7 (Fig. 3a), JIM5 (Fig. 3b) and 2F4 (Fig. 3c) Abs were observed. Weak fluorescence from JIM7 and JIM5 antibodies was also present in the walls of the generative and sterile cells as well as between the 


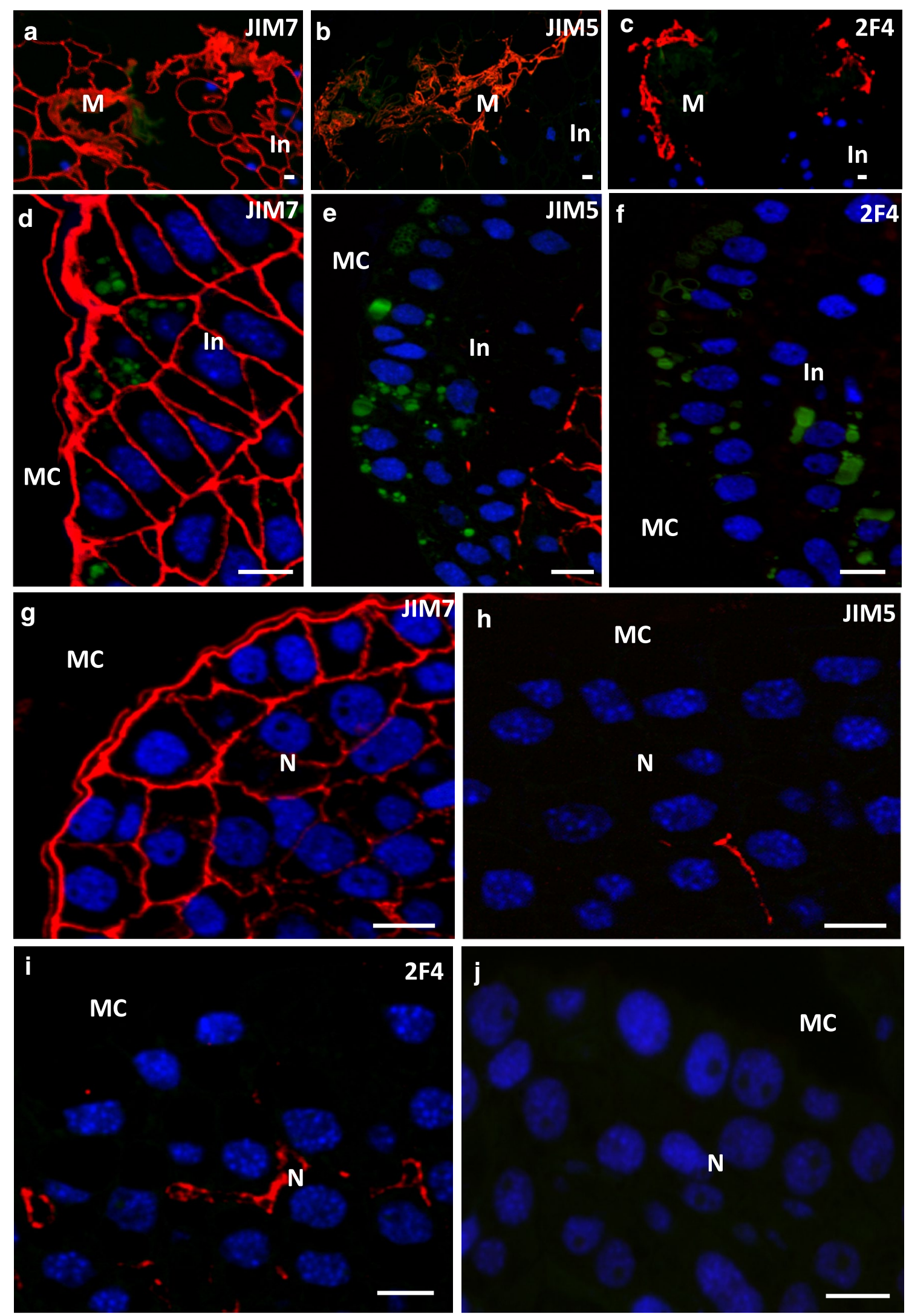


prothallial and sterile cells (Fig. 3a, b). In the pollen cell walls, labelling with 2F4 was not detected (Fig. 3c).

In the ecm of the unpollinated stigmatic tip, the main epitope found was that recognised by the JIM7 Ab (Fig. 4a). In this tissue, the JIM5 fluorescence was irregular and more intense on the stigmatic tip surface (Fig. 4b). Before pollination, fluorescence of the $2 \mathrm{~F} 4 \mathrm{Ab}$ was not detected (Fig. 4c).

Localisation of $\mathrm{HG}$ epitopes in the pollinated larch ovule

\section{Pollination stage}

Pollination induced changes in the distribution of HG epitopes in both the pollen grain and stigmatic tip. In the wall of the pollen grain adhering to the stigmatic tip, the level of fluorescence after labelling with the JIM7 Ab was significantly lower than before pollination (Fig. 4a, d). The strong labelling with the JIM5 Ab was localised to the pollen wall, and the fluorescence was visible as two layers (Fig. 4e). None of the HG epitopes were detected in the thin walls of the pollen cells (Fig. 4d, e). The fluorescence derived from the $2 \mathrm{~F} 4 \mathrm{Ab}$ was observed only in the pollen wall (Fig. 4f). After pollination, the epitope recognised by the $2 \mathrm{~F} 4 \mathrm{Ab}$ also appeared in the stigmatic tip but only at the place of pollen grain adhesion (Fig. 4f). The walls of the degenerating stigmatic tip cells were still labelled by JIM7 (Fig. 4d) and JIM5 (Fig. 4e) Abs. At this time, only the low-unesterified form of $\mathrm{HG}$ was detected in the integument (Fig. 4g-i).

\section{Pollen grains in the micropylar canal}

At the time of pollen grain engulfment into the micropylar canal, all analysed epitopes were detected in the material formed as a result of stigmatic tip degeneration (Fig. 5a-c). The epitope bound by JIM7 was mainly localised to the apoplast of the integument (Fig. 5d). Particularly, intense signal, sometimes observed as two layers, was observed in the integumentary wall coating the micropylar canal. Surface cells of the integument were not labelled with the JIM5 (Fig. 5e) or with 2F4 Abs (Fig. 5f). The epitope recognised by the JIM5 Ab was only detected in the walls of cells situated deep in the integument (Fig. 5e).

Immediately after the engulfment of pollen grains into the micropylar canal, the first changes in the distribution of the HG epitopes were observed in the nucellus. The walls of the nucellus cells were still labelled by the JIM7 $\mathrm{Ab}$ (Fig. 5g); however, for the first time, this tissue showed a weak labelling with the JIM5 (Fig. 5h) and 2F4 Abs (Fig. 5i). During gametophyte cellularisation and archegonia differentiation, the wall of the pollen grain present in the micropylar canal was devoid of exine. In this wall, the
JIM7 Ab labelling decreased further (Figs. 6a, 4d), and the JIM5 Ab labelling increased (Figs. 6b, 4e). The fluorescence indicating the presence of calcium cross-linked HG almost completely disappeared (Fig. 6d). In the area of the degenerating stigmatic tip, strong labelling with the JIM7 Ab was observed at the site of pollen grain adhesion and in the cell walls of the integument (Fig. 6a); however, the cell walls of the integument were not labelled by the JIM5 Ab (Fig. 6b).

The 2F4 Ab intensively labelled the apoplast coating the micropylar canal (Fig. 6c). In the ecm of the canal, the signal was observed as small sparse clusters. During ovule development, a decrease in the level of $2 \mathrm{~F} 4 \mathrm{Ab}$ labelling occurred in the inner surface of the integument and was accompanied by an increase in the number of fluorescence clusters in the micropylar canal (Fig. 6d). At this time, considerable changes in $\mathrm{HG}$ distribution were observed in the nucellus. In the ecm of the nucellus, all examined epitopes were present; however, their distribution was different. JIM7 Ab fluorescence was observed in both, the secretions present on the nucellar surface and in the walls of the epidermal and subepidermal cells (Fig. 6e). Epitope recognised by the JIM5 Ab was not detected in the apoplast of epidermal or subepidermal cells (Fig. 6f). However, the label was present in the ecm of cells situated deeper in the nucellus (Fig. 6f) and was visible as numerous, irregular clusters. Such a localisation pattern was characteristic for this period, as fluorescence was observed almost exclusively in the longitudinal cell walls of the nucellus (Fig. 6f). The epitopes recognised by $2 \mathrm{~F} 4$ were localised in the walls of the epidermal and subepidermal cells of the nucellus as well as in the secretions present on its surface (Fig. 6g).

Pollen grain germination and pollen tube growth-the stage of the mature ovule

At the time of archegonia maturity, germinating pollen grains and growing pollen tubes were visible on the nucellar surface. In the wall of the germinating pollen grains, a weak fluorescence from the JIM7 Ab was still observed (Fig. 7a). The JIM7 Ab labelling was also present in the pollen cytoplasm.

During pollen germination and pollen tube growth, a strong labelling of JIM7 Ab was still localised in the walls of the nucellar cells (Fig. 7a, b) as well as in the area of pollen tube growth (Fig. 7b). Epitopes recognised by JIM5 (Fig. 7c) and 2F4 Abs (Fig. 7d) were also detected in the area of pollen tube growth. The labelling from the JIM5 $\mathrm{Ab}$ (Fig. 7c) was intense and was found mainly around tip of the pollen tube; in contrast, the labelling of the $2 \mathrm{~F} 4$ $\mathrm{Ab}$ was present in the form of small clusters (Fig. 7d). By light microscopy, we could not clearly confirm whether the observed labelling was present in the pollen tube wall or only in the ecm of nucellus. For this reason, the pectin 

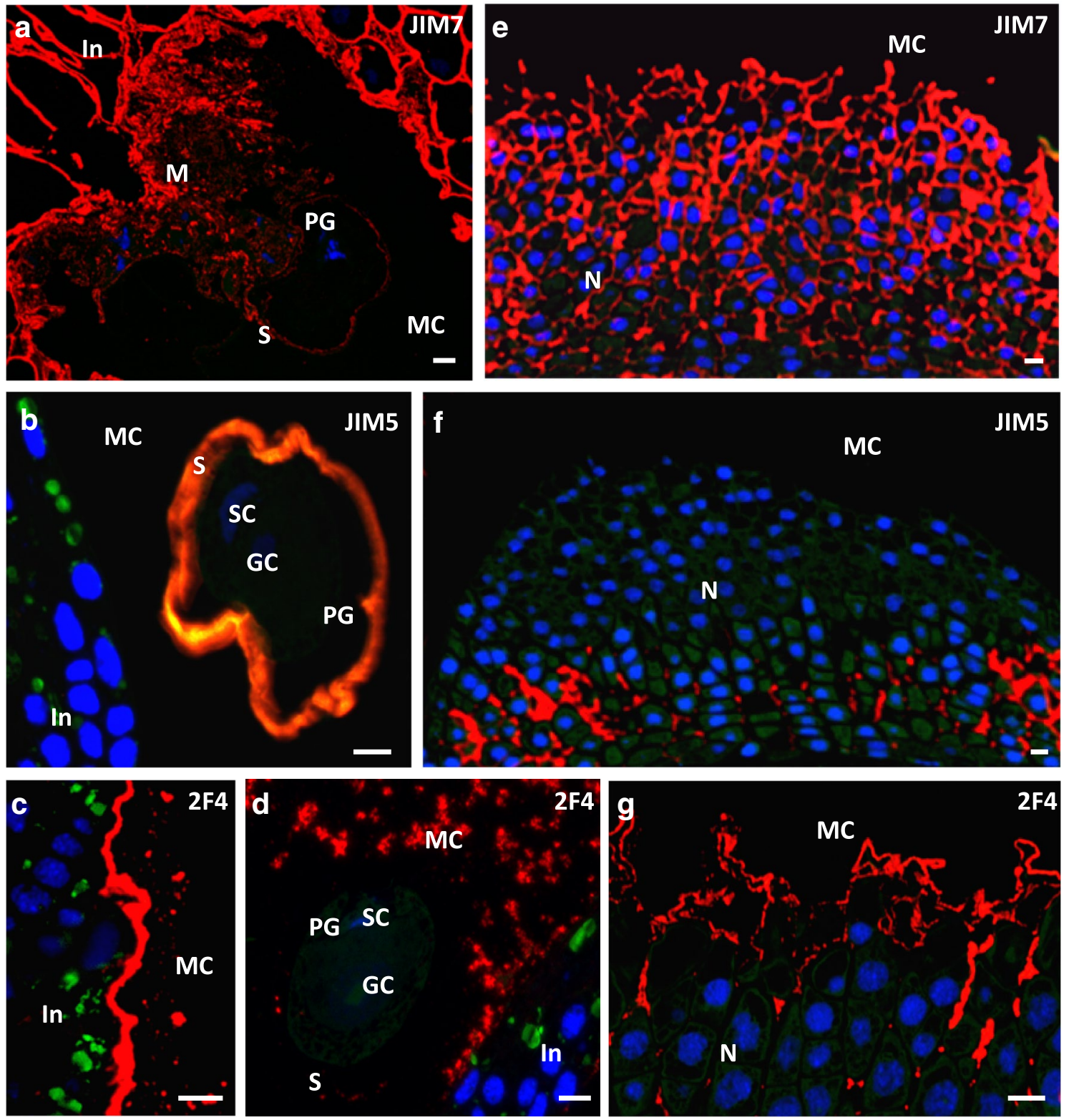

Fig. 6 Immunolocalisation of HG in the larch ovule after the engulfment of the pollen grain into the micropylar canal-cellular gametophyte stage. a-d The pollen grain in the micropylar canal. a The JIM7 fluorescence in the pollen grain wall was weak. Intense fluorescence was visible in the remnants of the degenerated cells of the stigmatic tip and in the walls of the integumentary cells. b The JIM5 labelling was localised to the pollen grain wall. The micropylar canal and apoplast of the integument were devoid of fluorescence. $\mathbf{c}$ The 2F4 signal shortly after engulfment of pollen grains was strong on the surface of canal, and clusters of fluorescence were also visible in the micropylar canal. d Later, the apoplast of the integument was not labelled by $2 \mathrm{~F} 4$. Numerous clusters of fluorescence were observed in the material surrounding the pollen grain. The pollen grain wall was almost completely devoid of the labelling. e-g The nucellus. e The JIM7 signal was found in all the cell walls and on the nucellar surface. $\mathbf{f}$ Single clusters of JIM5 fluorescence were observed in the proximal region of the nucellus. $\mathrm{g}$ The $2 \mathrm{~F} 4$ labelling was visible in the cell walls of the cells situated in the apical region of the nucellus and in the secretions on its surface. $G C$ generative cell, In integument, $M$ stigmatic tip, $M C$ micropylar canal, $N$ nucellus, $P G$ pollen grain, $S$ pollen wall, $S C$ sterile cell, $T C$ tube cell. Scale bars $10 \mu \mathrm{m}$

nucellus. In comparison with the earlier developmental stage, the fluorescence intensity after labelling with JIM5 (Fig. 7c, f) and 2F4 (Fig. 7d, g) was clearly lower. In the ecm of the nucellar apex that did not contain pollen grains, the signals from both JIM5 (Fig. 7f) and 2F4 Abs (Fig. 7g) composition of the pollen tube wall was studied in in vitro growing pollen tubes.

When mature archegonia were present in the ovule, considerable changes in the distribution pattern of low esterified and calcium cross-linked HG were observed in the 

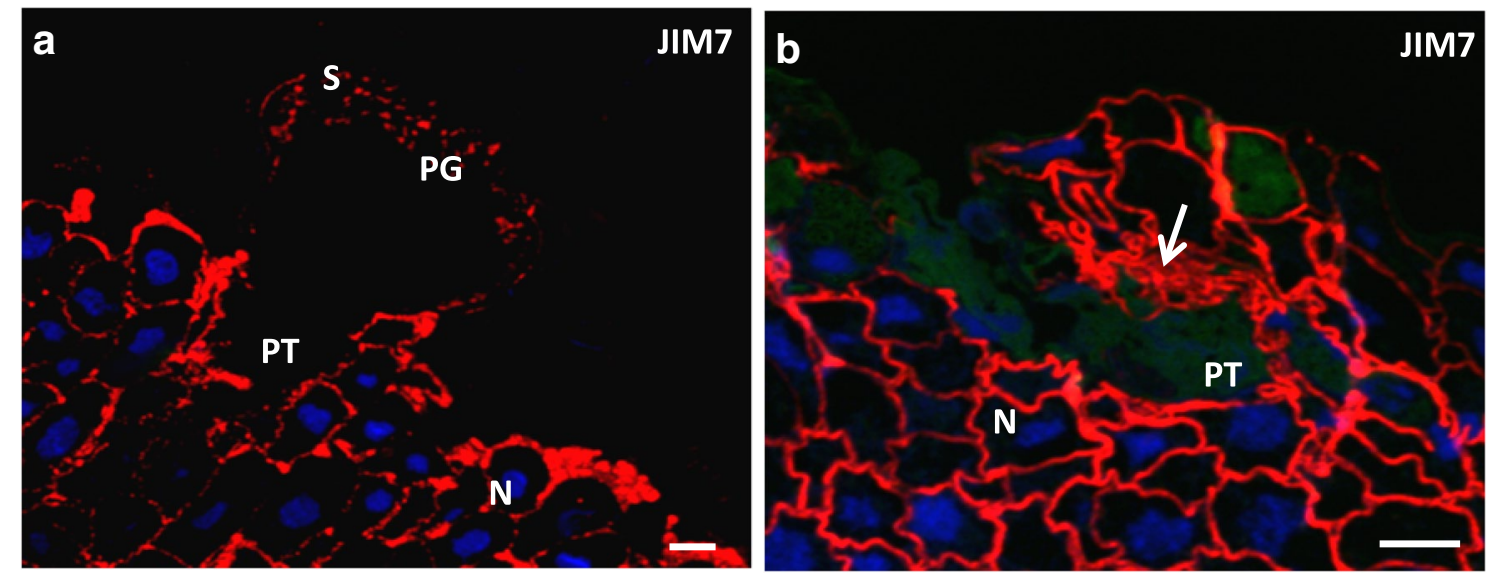

c

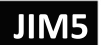

d

2F4
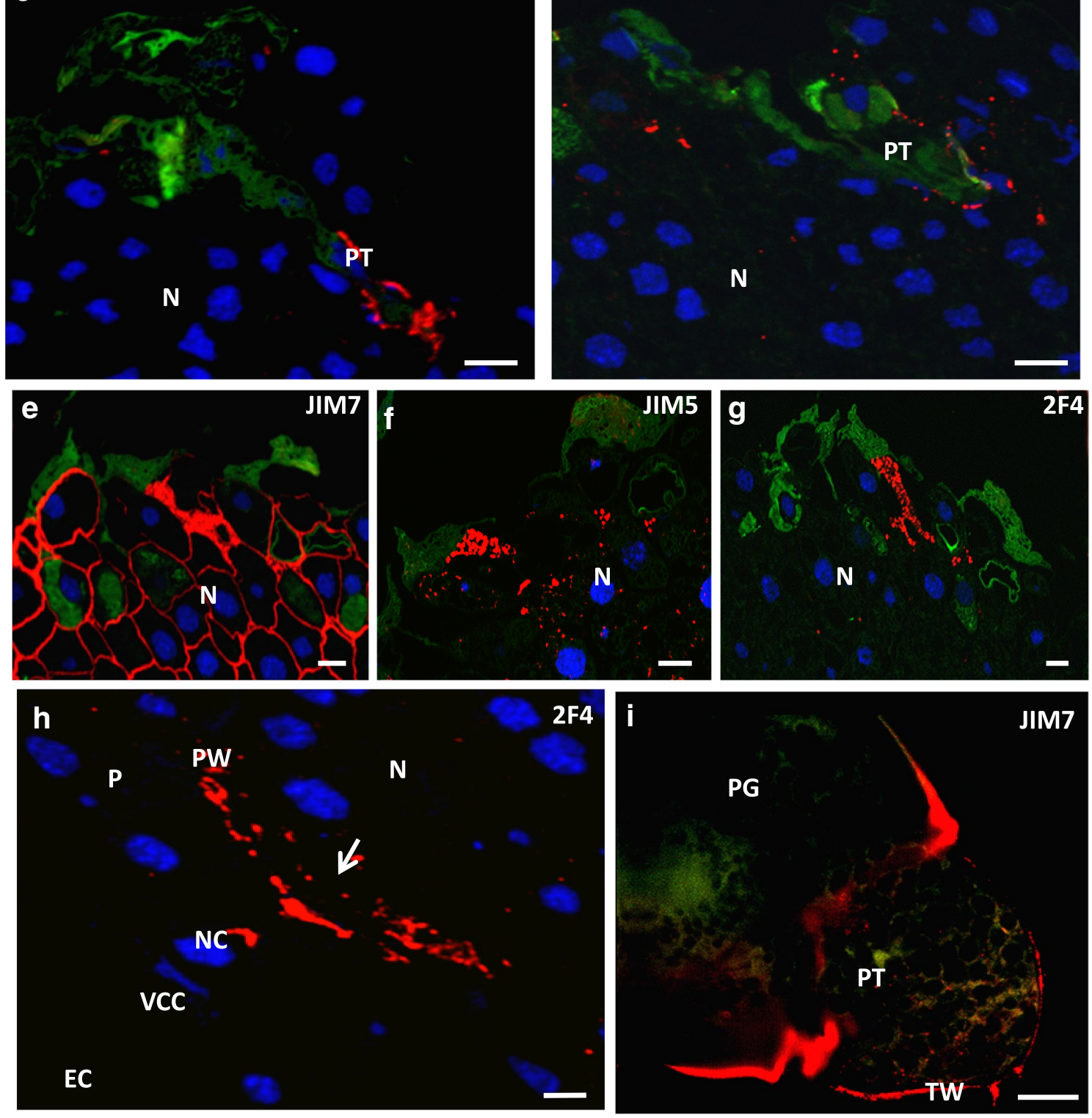

$\mathbf{N}$

PT

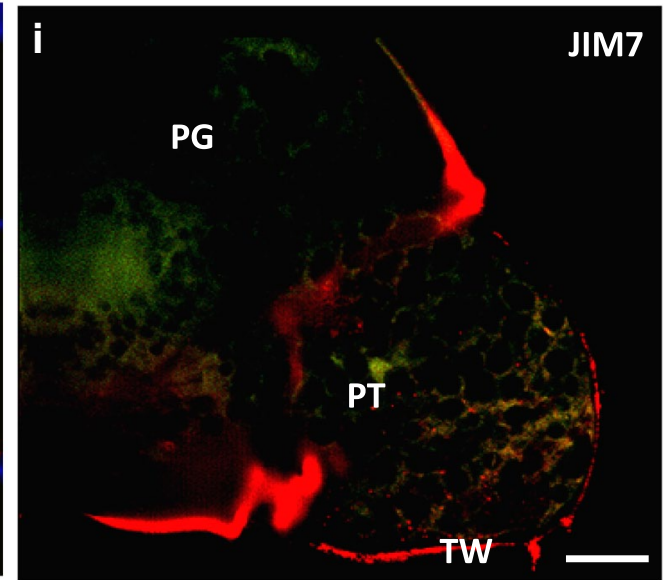


4Fig. 7 Immunolocalisation of HG in the mature ovule. a-d Pollen grain germination and pollen tube growth. a The JIM7 labelling was weak in the pollen grain wall and likely also in the pollen tube wall; small clusters of fluorescence were localised in the pollen cytoplasm. Intense labelling was visible in all the cell walls of the nucellar cells. b-d The site where the pollen tube overgrows the nucellus. b The JIM7 was present in the nucellar cell walls, and intense labelling was visible in the degenerated cells adjacent to the pollen tube (arrow). c Signal of JIM5 was seen only in the area of pollen tube growth. d The 2F4 labelling existed as single spots localised mainly in the area of pollen tube growth. e-g The nucellus of the mature ovule, the site where the pollen grain is not present. e The fluorescence from JIM7 was present in the cell walls, but labelling was not visible on the nucellar surface. f, g Signal from JIM5 (f) and 2F4 (g) was detected only in the apical region of the nucellus. $\mathbf{h}$ The area between the nucellus and prothallium. The fluorescence from $2 \mathrm{~F} 4$ was localised only in the wall separating the prothallium from the nucellar cells. $\mathbf{i}$ Immunolocalisation of JIM7 in the in vitro growing larch pollen tube. The labelling was strong in the intine surrounding the germinating pollen tube. In the pollen tube, the fluorescence was visible in the wall and in small clusters in cytoplasm. $E C$ egg cell, $N$ nucellus, $N C$ neck cell, $n u$ nucleus, $P$ prothallium, $P G$ pollen grain, $P T$ pollen tube, $P W$ prothallial wall, $S$ pollen wall, $T C$ tube cell, $V C C$ ventral canal cell, $T W$ tube wall. Scale bars $10 \mu \mathrm{m}$

were distributed mainly as single fluorescence clusters of different sizes, and accumulation of esterified HG was also observed (Fig. 7e). At this time, the 2F4 Ab labelling was also present at the top of the archegonium over the neck cells (Fig. 7h). Our earlier investigations have demonstrated that HGs recognised by JIM7 and JIM5 were also localised in this area (Rafińska and Bednarska 2011).
Immunocytochemical localisation of $\mathrm{HG}$ in in vitro germinating pollen grains revealed the presence of only highesterified HG (Fig. 7i). Accumulation of epitope recognised by the JIM7 Ab was observed in the intine around the site of pollen tube germination. In the pollen tube, labelling was present in the cell wall as well as in the cytoplasm, where it was visible in the form a small clusters. No low methyl-esterified or $\mathrm{Ca}^{2+}$-associated HGs were detected in the pollen grain or pollen tube (data not shown).

Temporal and spatial changes in the distribution of the different categories of HG during male gametophyte-ovule interaction in L. decidua are summarised in Fig. 8.

\section{Discussion}

Pollen grain adhesion and its transfer to the micropylar canal

In the apoplast of the unpollinated stigmatic tip of $L$. decidua as well as in the stigmas of angiosperms (Bednarska et al. 2005; Sage et al. 2009), both high methyl-esterified and low methyl-esterified HG were localised. In this study, we showed that pollination induced changes in the pectin composition of the stigmatic tip, especially at the site of pollen grain adhesion where calcium cross-linked HG appeared. The presence of this category of HG at the site of physical contact between the ecm of the stigmatic

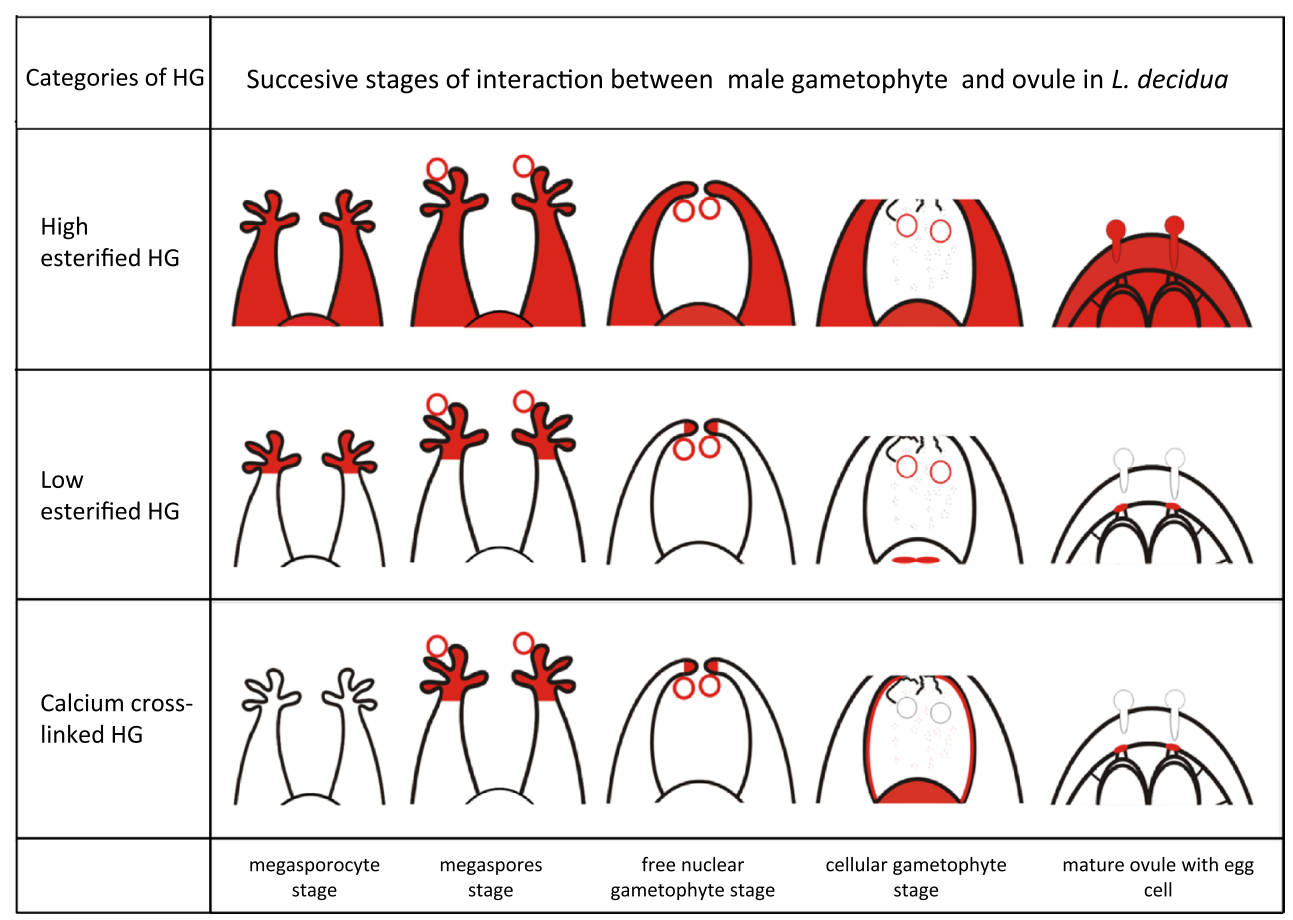

Fig. 8 Schematic localisation of different categories of HG (red colour) in the male gametophyte and the ovule during successive stages of their interaction in L. decidua 
tip and the pollen wall suggests that these molecules play an important role during the early interaction steps between the male gametophyte and the ovule in larch plants. Many reports have suggested that in angiosperms, low-esterified HG is responsible for the adhesion of the pollen grain to the stigma and that it most likely occurs by calcium crosslinking (Mollet et al. 2000). Here, we showed that in $L$. decidua, calcium cross-linked HG is involved in the adhesion between the pollen grain and stigmatic tip.

After a few days of pollination, the stigmatic tip and attached pollen grains were drawn into the micropylar canal. At this time, changes in the HG composition of the pollen grain cell wall were observed. The lack of calcium cross-linked HG indicates its degradation in the pollen wall during the pre-germinative period. Lysis of pectin is performed by polygalacturonase (Bonnin et al. 2002; Wolf et al. 2009). The accessibility of polygalacturonase to lowesterified HG, the substrate for this enzyme, requires disintegration of the 'egg-box' structure. Thus, it is probable that after entry of the pollen grains into the micropylar canal, dissociation of links between HG chains in the stigmatic tip cell as well as in the pollen wall occurs. We postulate that the disintegration of the 'egg-box' structure leads to the disappearance of adhesion between the male gametophyte and the stigmatic tip and, thus, allows the transfer of pollen grains to the nucellus.

In the pollen wall, significant levels of low methylesterified HG indicate the presence of large amounts of negatively charged COO-groups. It is known that their presence is associated with cell wall hydration and swelling (Zsivanovits et al. 2004). It is possible that swelling of low methyl-esterified HG, present in the pollen wall, causes exine rupture and shedding.

Moreover, 'egg-box' disintegration leads to the appearance of the free calcium ions in the micropylar canal. We propose that this pool of calcium ions can subsequently be bound by the HG, which is present in ECM of the micropylar canal. We have found that the presence of pollen in the micropylar canal induced changes in the pectin composition of its ecm. Calcium cross-linked HG appeared in the walls coating the micropylar canal and as single, irregular clusters in the canal secretion. This suggests that this category of HG is a component of the secretion that is necessary for the transfer of pollen grains to the nucellar apex.

\section{Pollen germination and pollen tube growth}

Nucellar surface cells are secretory and degenerate during the transfer of pollen grains through the micropylar canal. At this time, the changes in the nucellus HG seemed to reflect the preparation of the environment for pollen germination and tube growth. During pollination, the nucellar cell walls showed only the presence of esterified HG.
Our results suggest that when pollen was present in the micropylar canal, deesterification of $\mathrm{HG}$ and $\mathrm{Ca}^{2+}$ binding occurred in the ecm of the nucellus. Similarly, in angiosperm plants, pollination induced deesterification of HG present in the extracellular matrix of the style transmitting tissue (Lenartowska et al. 2001). A decrease in the level of deesterified and $\mathrm{Ca}^{2+}$-associated $\mathrm{HG}$ was observed in the nucellus during pollen grain germination and tube growth. Both these categories of $\mathrm{HG}$ were most likely degraded. Lysis of calcium cross-linked $\mathrm{HG}$ leads to $\mathrm{Ca}^{2+}$ release. Thus, we postulate that the precisely regulated degradation of this HG creates an optimal calcium environment for pollen grain germination and pollen tube growth. Many studies in both angiosperms and gymnosperms showed that $\mathrm{Ca}^{2+}$ plays a particularly important role in pollen grain germination and pollen tube growth (Malhó et al. 2000; Fernando et al. 2005; Lazzaro et al. 2005; Wu et al. 2008). Both lysis of deesterified HG (Lenartowska et al. 2001) and a strong increase in the $\mathrm{Ca}^{2+}$ level in the extracellular matrix of the pollinated solid style have been described previously in some angiosperms (Lenartowska et al. 1997). Thus, the mechanism of creating an optimal $\mathrm{Ca}^{2+}$ environment for pollen tube growth in the nucellus of the $L$. decidua ovule seems to be similar to that observed in the solid style of flowering plants. An additional aspect of HG lysis in larch plants is cell wall loosening, which facilitates penetration of this tissue by growing pollen tubes.

Characteristic accumulation of calcium cross-linked HG was visible above neck cells of the mature archegonium. Our recent investigations have shown that in this area, both high methyl-esterified HG and low methyl-esterified HG are also present (Rafińska and Bednarska 2011). The specific pectin composition of these cell walls indicates that this region could be involved in attracting and directing the pollen tube. In angiosperms, these functions are performed by the filiform apparatus of the synergids, where elevated levels of pectins were also observed (see the review by $\mathrm{Li}$ et al. 2009).

In the cell wall of germinating pollen grains high-esterified HG was still present at a low level. However, the lack of low methyl-esterified $\mathrm{HG}$ indicates that it undergoes lysis. At this time, high methyl-esterified HG was also present in the pollen cytoplasm, which most likely reflects its synthesis for the growing pollen tube. In gymnosperms, similar to angiosperms, HG is an essential component of the expanding pollen tube wall (Derksen et al. 1999; Fernando et al. 2005; Parre and Geitmann 2005; Chen et al. 2008; Wu et al. 2008; Dardelle et al. 2010; Lehner et al. 2010). In vivo, it was difficult to distinguish the wall of pollen tubes from the walls of the adjacent nucellar cells. At the border between the pollen tube and the nucellus, both low-esterified and calcium cross-linked HG were observed. 
In the wall of $L$. decidua pollen tubes growing in vitro, only high methyl-esterified HG was localised, and no low methyl-esterified or $\mathrm{Ca}^{2+}$-associated $\mathrm{HG}$ was present. The punctate localisation of high methyl-esterified HG in the tube cytoplasm may indicate that its synthesis and secretion takes place during pollen tube growth. It should be noted that in vitro growing pollen tubes were very short, and the HG composition of their walls may not reflect the in vivo state. High methyl-esterified, but not deesterified, HG was detected in in vitro growing pollen tubes of Pinus sylvestris and Pinus densiflora (Derksen et al. 1999; Mogami et al. 1999). However, low methyl-esterified HG was detected in the walls of Picea wilsonii, P. meyeri and $P$. bungeana pollen tubes (Chen et al. 2008, 2009; Wu et al. 2008).

Low methyl-esterified HG is a main component of the angiosperm pollen tube wall. It is suggested that in the pollen tube wall, the negative charges of deesterified $\mathrm{HG}$ bind $\mathrm{Ca}^{2+}$, which imparts structural rigidity to the cell wall (Hepler and Winship 2010). Low methyl-esterified HG in pollen tubes of angiosperms is derived from successive deesterification of esterified HG originally deposited in the tip. The obtained results and literature data suggest that in the pollen tubes of some gymnosperms, this process does not occur (L. decidua, P. sylvestris and P. densiflora). It is possible that the shorter and much slower growing tubes of gymnosperms do not need mechanical support in the form of deesterified HG.

In summary, our investigations suggest that in the male gametophyte-ovule interaction in L. decidua, low methylesterified and calcium cross-linked HG play an important role (Fig. 8). HG was only deesterified in the cell walls and extracellular matrix of the ovule tissues that interact with male gametophyte, and a pool of low methyl-esterified HG was cross-linked by $\mathrm{Ca}^{2+}$. This last category of $\mathrm{HG}$ is most likely involved in adhesion between the pollen and the stigmatic tip and may be a reservoir of $\mathrm{Ca}^{2+}$ in ecm of the ovule. The precisely regulation of calcium cross-linked HG degradation might provide an optimal $\mathrm{Ca}^{2+}$ environment for pollen grain germination and pollen tube growth.

Acknowledgments We would like to thank K. Zienkiewicz (Department of Cell Biology, Nicolaus Copernicus University, Toruń, Poland, Spanish National Research Council Experimental Station of Zaidín, Madrid, Spain) for helpful comments and proofreading of the manuscript. This work was supported by European Social Fund and Kujawsko-Pomorski Province Council project 'Stypendia dla doktorantów 2008/2009-ZPORR' and Nicolaus Copernicus University Grant 304-B.

Open Access This article is distributed under the terms of the Creative Commons Attribution License which permits any use, distribution, and reproduction in any medium, provided the original author(s) and the source are credited.

\section{References}

Bednarska E, Lenartowska M, Niekraś L (2005) Localization of pectins and $\mathrm{Ca}^{2+}$ ions in unpollinated and pollinated wet (Petunia hybrida Hort.) and dry (Haemanthus albiflos L.) stigma. Folia Histochem Cytobiol 43:249-259

Bonnin E, Le Goff A, KÖrner R, Vigoroux J, Roepstorff P, Thibault JF (2002) Hydrolysis of pectins with different degrees and patterns of methylation by the endopolygalacturonase of Fusrium moniliforme. Biochim Biophys Acta 1596:83-94

Chen KM, Wu GL, Wang YH, Tian CT, Šamaj J, Baluška F, Lin JX (2008) The block of intracellular calcium release affects the pollen tube development of Picea wilsonii by changing the deposition of cell wall components. Protoplasma 233:39-49

Chen T, Wu X, Chen Y, Li X, Huang M, Zheng M, Baluška F, Šamaj J, Lin J (2009) Combined proteomic and cytological analysis of $\mathrm{Ca}^{2+}$-calmodulin regulation in Picea meyeri pollen tube growth. Plant Physiol 149:1111-1126

Cheung AY, Wang H, Wu HM (1995) A floral transmitting tissuespecific glycoprotein attracts pollen tubes and stimulates their growth. Cell 82:383-393

Clausen MH, Willats WG, Knox JP (2003) Synthetic methyl hexagalacturonate hapten inhibitors of anti-homogalacturonan monoclonal antibodies LM7, JIM5 and JIM7. Carbohydr Res 338:1797-1800

Coimbra S, Almeida J, Junqueira V, Costa ML, Pereira LG (2007) Arabinogalactan proteins as molecular markers in Arabidopsis thaliana sexual reproduction. J Exp Bot 58:4027-4035

Costa M, Pereira AM, Rudall PJ, Coimbra S (2013) Immunolocalization of arabinogalactan proteins (AGPs) in reproductive structures of an early-divergent angiosperm, Trithuria (Hydatellaceae). Ann Bot 111:183-190

Dardelle F, Lehner A, Ramdani Y, Bardor M, Lerouge P, Driouich A, Mollet JC (2010) Biochemical and immunocytological characterization of Arabidopsis pollen tube cell wall. Plant Physiol 153:1563-1576

Derksen J, Li Y-Q, Knuiman B, Geurts H (1999) The wall of Pinus sylvestris L. pollen tubes. Protoplasma 208:26-36

Fernando DD, Lazzaro MD, Owens JN (2005) Growth and development of conifer pollen tubes. Sex Plant Reprod 18:149-162

Ge LL, Tian HQ, Russell SD (2007) Calcium function and distribution during fertilization in angiosperms. Am J Bot 94:1046-1060

Ge LL, Xie CT, Tian HQ, Russell SD (2009) Distribution of calcium in the stigma and style of tobacco during pollen germination and tube elongation. Sex Plant Reprod 22:87-96

Hepler PK, Winship LJ (2010) Calcium at the cell wall-cytoplast interface. J Integr Plant Biol 52:147-160

Hristova K, Lam M, Feild T, Sage TL (2005) Transmitting tissue ECM distribution and composition, and pollen germinability in Sarcandra glabra and Chloranthus japonicus (Chloranthaceae). Ann Bot 96:779-791

Jauh GY, Lord EM (1996) Localization of pectins and arabinogalactan-proteins in lily (Lilium longiflorum L.) pollen tube and style, and their possible roles in pollination. Planta 199:251-261

Knox JP, Linstead PJ, King J, Cooper C, Roberts K (1990) Pectin esterification is spatially regulated both within cell walls and between developing tissues of root apices. Planta 181:512-521

Lazzaro MD, Cardenas L, Bhatt AP, Justus CD, Phillips MS, Holdaway-Clarke TL, Hepler PK (2005) Calcium gradients in conifer pollen tubes; dynamic properties differ from those seen in angiosperms. J Exp Bot 56:2619-2628

Lehner A, Dardelle F, Soret-Morvan O, Lerouge P, Driouich A, Mollet JC (2010) Pectins in the cell wall of Arabidopsis thaliana pollen tube and pistil. Plant Signal Behav 5:1282-1285 
Lenartowska M, Bednarska E, Butowt R (1997) $\mathrm{Ca}^{2+}$ in the pistil of Petunia hybrida Hort. during growth of the pollen tubecytochemical and radiographic studies. Acta Biol Cracov Bot 39:79-89

Lenartowska M, Rodrigue-Garcia MI, Bednarska E (2001) Immunocytochemical localization of esterified and unesterified pectins in unpollinated and pollinated styles of Petunia hybrid Hort. Planta 213:182-191

Lenartowska M, Krzesłowska M, Bednarska E (2011) Pectin dynamic and distribution of exchangeable $\mathrm{Ca}^{2+}$ in Haemanthus albiflos hollow style during pollen-pistil interactions. Protoplasma 248:695-705

Li DX, Lin MZ, Wang YY, Tian HQ (2009) Synergid: a key link in fertilization of angiosperms. Biol Plant 53:401-407

Liners F, van Custem P (1992) Distribution of pectic polysaccharides throughout walls of suspension-cultured carrot cells: an immunocytochemical study. Protoplasma 170:10-21

Liners F, Letesson JJ, Didembourg C, van Custem P (1989) Monoclonal antibodies against pectin: recognition of a conformation induced by calcium. Plant Physiol 91:1419-1424

Liners F, Thibault JF, van Cutsem P (1992) Influence of the degree of polymerization of oligogalacturonates and of esterification pattern of pectin on their recognition by monoclonal antibodies. Plant Physiol 99:1099-1104

Malhó R, Camacho L, Moutinho A (2000) Signalling pathways in pollen tube growth and reorientation. Ann Bot 85:59-68

Mogami N, Nakamura S, Nakamura N (1999) Immunolocalization of the cell wall components in Pinus densiflora pollen. Protoplasma 206:1-10

Mollet J-C, Park S-Y, Nothnagel EA, Lord EM (2000) A lily stylar pectin is necessary for pollen tube adhesion to an in vitro stylar matrix. Plant Cell 12:1737-1757

Park SY, Jauh G-Y, Mollet J-C, Eckard KJ, Nothnagel EA, Walling LL, Lord EM (2000) A lipid transfer-like protein is necessary for lily pollen tube adhesion to an in vitro stylar matrix. Plant Cell 12:151-163

Parre E, Geitmann A (2005) Pectin and the role of the physical properties of the cell wall in pollen tube growth of Solanum chacoense. Planta 220:582-592
Rafińska K, Bednarska E (2011) Localisation pattern of homogalacturonan and arabinogalactan proteins in developing ovules of the gymnosperm plant Larix decidua Mill. Sex Plant Reprod $24: 75-87$

Sage TL, Hristova-Sarkovski K, Koehl V, Lyew J, Pontieri V, Bernhardt P, Weston P, Bagha S, Chiu G (2009) Transmitting tissue architecture in basal-relictual angiosperms: implications for transmitting tissue origins. Am J Bot 96:183-206

Suárez C, Zienkiewicz A, Castro AJ, Zienkiewicz K, MajewskaSawka A, Rodríguez-García MI (2013) Cellular localization and levels of pectins and arabinogalactan proteins in olive (Olea europaea L.) pistil tissues during development: implications for pollen-pistil interaction. Planta 237:305-319

Verhertruggen Y, Marcus SE, Haeger A, Ordaz-Ortiz JJ, Knox JP (2009) An extended set of monoclonal antibodies to pectic homogalacturonan. Carbohydr Res 344:1858-1862

Willats WGT, Limberg G, Buchholt HC, van Alebeek GJ, Benen J, Christensen TMIE, Visser J, Voragen A, Mikkelsen JD, Knox JP (2000) Analysis of pectic epitopes recognized by hybridoma and phage display monoclonal antibodies using defined oligosaccharides, polysaccharides and enzymatic degradation. Carbohydr Res 327:309-320

Wolf S, Mouille G, Pelloux J (2009) Homogalacturonan methyl-esterification and plant development. Mol Plant 2:851-860

Wu X, Chen T, Zheng M, Chen Y, Teng N, Šamaj J, Baluška F, Lin $\mathrm{J}$ (2008) Integrative proteomic and cytological analysis of the effects of extracellular $\mathrm{Ca}^{2+}$ influx on Pinus bungeana pollen tube development. J Proteome Res 7:4299-4312

Zhao J, Yang HY, Lord EM (2004) Calcium levels increase in the lily stylar transmitting tract after pollination. Sex Plant Reprod 16:259-263

Zsivanovits G, MacDougall AJ, Smith AC, Ring SG (2004) Material properties of concentrated pectin networks. Carbohydr Res 339:1317-1322 